\title{
Medication resistant tuberculosis: multi drug- resistant and extensively drug resistant
}

\begin{abstract}
Medication-resistant tuberculosis is a considerable across-the-board public health challenge that menace's the substantial advance made in tuberculosis heedfulness and precluding in current decades. Multidrug-resistant tuberculosis is caused by organisms that are resistant to the consummate effective anti-tuberculosis medications (isoniazid and rifampicin). Tuberculosis organisms resistant to the antibiotics used in its treatment are extendedly and happen in entire countries studied. Medication resistance noticed as a sequence of insufficient treatment and once tuberculosis organisms obtain resistance they can disseminate from person to person in the similar way as medication-sensitive tuberculosis. Multidrug-resistant tuberculosis sequences from either infection with organisms which are previously medication-resistant or perhaps advance in the program of a patient's treatment. Rifampicin-resistant tuberculosis is caused by bacteria that do not answered to rifampicin, one of the consummate influential anti- tuberculosis medications. These patients necessitated multidrug-resistant tuberculosis treatment. Extendedly medication-resistant tuberculosis is a figure of tuberculosis caused by organisms that are resistant to isoniazid and rifampicin (i.e. multidrug-resistant tuberculosis) as well as every fluoroquinolone and any of the second-line anti- tuberculosis injectable drugs (amikacin, kanamycin or capreomycin). Extendedly medication-resistant tuberculosis can elaborate when second-line medications are used incorrectly or wrongly managed and upon become ineffective.
\end{abstract}

Keywords: tuberculosis, multidrug-resistant tuberculosis, extensively drug-resistant tuberculosis
Volume 8 Issue 4 - 2021

\author{
Gudisa Bereda \\ Department of Pharmacy, Negelle Health Science College, \\ Ethiopia
}

Correspondence: Gudisa Bereda, Department of Pharmacy, Negelle Health Science College, Guji, Ethiopia, $\mathrm{Tel}+251913118492 /+251919622717$

Email gudisabareda95@gmail.com

Received: October 25, 2021 | Published: December 23, 202
Abbreviations: AMR, antimicrobial resistance; Bdq, bedaquiline; Cfz, Clofazimine; TB, tuberculosis; DOTS, directly observed treatment short-course; DR, drug-resistant; EMB, ethambutol; E, Ethambutol; $\mathrm{H}^{\mathrm{H}}$, high dose isoniazid; HIV, human immune virus; INH, isoniazid; Lfx, Levofloxacin; LTBI, latent TB infection; MAC, mycobacterium avium complex; Mfx, moxifloxacin; MDR-TB, multi drug-resistant tuberculosis; NTB, national tuberculosis programme; PZA, pyrazinamide; Pto, prothionamide; RIF, Rifampin; RR-TB, rifampicin-resistant tuberculosis; SSA, subsaharan africa; XDR-TB, extensively drug resistance tuberculosis; Z: pyrazinamide

\section{Introduction}

WHO recommended six-month criteria program of medicine, copious countries treat TB infirmity using four $1^{\text {st }}$-line (RIF, INH, PZA and EMB) anti-TB medications. ${ }^{1}$ The WHO divides antiTB drugs into 5 groups: (1) $1^{\text {st }}-$ line drugs; (2) FQ (ofloxacin, levofloxacin, moxifloxacin); (3) injectables (kanamycin, amikacin, capreomycin, streptomycin); (4) oral bacteriostatic second- line drugs (cycloserine/terizidone, ethionamide/prothionamide, PAS); and (5) anti-TB medications with limited data on efficacy: clofazimine (Cfz), linezolid (Lzd), amoxicillin/ clavulanate (amoxi-clav), carbapenems (imipenem-cilastatin and meropenem) high- dose $\mathrm{H}$, bedaquiline (Bdq) and delamanid (the latter two have been approved for a 6-month course in pre-XDR/XDR-TB). Medications in from group II to IV (except streptomycin) are considered $2^{\text {nd }}-$ line therapy, while group $\mathrm{V}$ drugs are also known as $3^{\text {rd }}$-line therapy. ${ }^{2,3}$ Medication-resistant TB is segment of the expanding knot of antimicrobial-resistant superbugs that do not answered to existing medicines, sequencing in smaller treatment alternatives and escalating mortality rates for infirmities that would frequently be healable enclosing TB. Across-the-board advancement colleague must move speedy to enclose this menace of AMR before it escalates to claim millions of lives around the world..$^{4-8}$ TB patients frequently stop treatment previously it is comprehensive, leading to medication resistant TB. ${ }^{9}$ Medication-resistant TB infirmity can advance in dual distinctive ways, called primary and secondary resistance. Primary resistance happens in persons who are firstly exposed to and infected with resistant organisms or medicine resistance amid fresh cases i.e. persons who have never been previously treated for TB. Secondary resistance, or acquired resistance, progresses during TB therapy, either because of the patient was treated with an inadequate regimen or because the patient did not take the prescribed regimen appropriately or because of other conditions such as drug malabsorption or drug-drug interactions leading to low serum levels or medicine resistance amid previously treated individuals. ${ }^{10}$

\section{Prevalence of MDR-TB and XDR-TB medication- resistant}

TB was the cause of 1.7 million deaths in 2009 , highly amid people in their consummate formative years. There are 9.4 million fresh infections each year. MDR-TB and XDR-TB have become further dominant in the last 15-20 years. Both MDR- and XDRTB are pretense durable complaint in diagnosis and treatment. ${ }^{11,12}$ The year 2019 WHO narrate displays in 2018, there were around half a million fresh cases of RR-TB (of which 78\% had multi MDR-TB, and multiplex cases are advancing XDR-TB amid re-treatment cases round the world. ${ }^{13}$ Across-the board, the WHO narrates an assessed frequency of $3.6 \%$ and $20.2 \%$ amid notified TB cases for preliminary and obtained MDR-TB respectively, with substantial country and regional differences.$^{14}$ Contempt the great load of TB in SSA supplied by HIV medication resistance supervision has not been broadly done, with solely 22 of the 46 countries narrating medication resistance 
document by 2005 . These surveys have been blueprinted to settle a nationwide MDR-TB frequency solely, and consummate of them had less sample sizes to ascertain otherness's between subpopulations or distinguish implicit pitfall factors of the prevalence of medication resistance. ${ }^{15-17}$

\section{Factors making MDR-TB and XDR-TB medication- resistant}

In a patient with active TB malady, factors that make or elaborate medication resistance enclose: The patient perhaps not take entire the medications prescribed, owing to every of the pursuing factors: dearth of resources, dogmatism/toxicity, fail to understanding well, discontinued medication furnish, distrust in the diagnosis, distrust in the efficacy or must-have of the treatment, jumble lifestyle; material abuse, socio-cultural outcomes, pregnancy, neuropsychiatric malady, there perhaps a dispensing or administration erroneous respecting the right dose, the patient perhaps not be prescribed the applicable dose, the patient perhaps not absorb the complete dose of medicine and/or have infirmity in demesnes where the discernment of lone or further of the medications perhaps be harmed, the furnisher perhaps not prescribe an tolerable TB regimen, the patient's organism perhaps previously be resistant to lone of the TB medications prescribed, leaving an unrecognized suboptimal TB regimen, the patient may have been inaccurately diagnosed as having LTBI, rather than active TB malady, and treated with monotherapy, the TB patient perhaps be taking therapy for disparate infirmity. That therapy perhaps coincidently enclose a lone medication active fight TB (rifabutin in an HIV patient for MAC prophylaxis; a fluoroquinolone for communityacquired pneumonia), the patient perhaps take TB medications without a prescription (occasionally applicable OTC exterior the US, or if taking medicines belonging to somebody else), The TB medications perhaps interact with disparate medications being taken by the patient. ${ }^{18-23}$ Treatment failure happens when medications are incorrectly prescribed owing to dearth of furnisher wisdom or owing to medications scarceness. Occasionally the patients perhaps close out $1^{\text {st }}$ line treatment owing to weaken side effects, dearth of an assistance network, incapability to withdraw time from function to obtain the treatment $\mathrm{s} /$ he necessitates or disparate rationale. Contradictory to what multitude people credent, it is seldom for medication resistance to be caused by the eager ignorance of patients to contend treatment. Factors such as non-adherence to prescribed medicine by the patient, physician erroneous consociated with scarce or malapropos chemotherapy prescribed, and deficiently working NTP consociated with meager medication fantabulous, dearth of DOTS and aberrational medication provision have been consociated with the frequency of MDR/RR-TB in multiplex ambient. ${ }^{24-26}$ Medication-resistant TB (MDR or XDR) is consummate ubiquitous in people who: do not take their TB medicine according to their program, do not take entire of their TB medications as prescribed by their doctor, advance TB malady de novo, after having taken TB medication in the past, come from areas of the world where drug resistant TB is ubiquitous, have exhausted time with somebody understood to have medicationresistant TB malady. ${ }^{27-29}$

\section{Multidrug-resistant tuberculosis}

MDR-TB is delineated as resistance to dual of the consummate substantial and effective " 1 st-line" medications, rifampicin and isoniazid, which are the preferable choice for treatment. 3.3\% of entire TB infirmity cases are MDR-TB. ${ }^{30}$ MDR-TB must be treated with socalled " 2 nd-line" medications which are least effective, further costly, and consociated with greater solemn side effects than $1^{\text {st }}$ line treatments.
Diagnosis of medication resistance is sophisticated, specifically in less resource countries; diagnosis perhaps take anyplace from 6 to 16 weeks and necessitates difficulty lab instrument. ${ }^{31-35}$ MDR-TB is TB owing to organisms which reveal great-level resistance to both isoniazid and rifampicin, with or without resistance to disparate antiTB medications. The molecular basis of resistance to isoniazid and rifampicin (and certain distinctive medications) is recently highly known. Resistance to isoniazid owes to change in genes of M.TB at one of dual foremost sites, in either the katG or inhA genes. ${ }^{36,37}$ The line of the challenge of MDR-TB has been demonstrated by the WHO in cross-sectional studies of medication resistance in either clinical solemn or whole-country cohorts. ${ }^{38}$ The current regimen for adults with MDR-TB treatment is 4-6 Bdq -Mfx-Pto-Cfz-Z-H ${ }^{\mathrm{H}}$-E / 5 LfxCfz-Z-E.

\section{Extensively drug resistance tuberculosis}

XDR-TB is delineated as MDR-TB that also does not answered to multiplex $2^{\text {nd }}$-line medications. It is measured that $5 \%$ of MDR-TB cases are XDR-TB. XDR-TB must be treated with uniform greater costly and toxic $3^{\text {rd }}$-line medications, and a program of treatment must be particularly customized to individual TB samples. Consummate patients with XDR-TB will die previously such estimation's can be going on, in higher segment owing to the sophistication of diagnosing the resistance in time. ${ }^{39,40}$ XDR-TB can advance when these $2^{\text {nd }}$ line medications are also used wrongly or managed incorrectly and thereupon also become ineffective. Because XDR-TB is resistant to $1^{\text {st }}$-line and $2^{\text {nd }}$-line medications, treatment alternatives are solemn restricted. It is thereupon crucial that TB regulate is controlled appropriately. ${ }^{41-43}$ XDR-TB can advance when $2^{\text {nd }}-$ line medications are used wrongly or managed incorrectly and thereupon become ineffective. ${ }^{44,45}$ This occurs when TB regulate programmers are deficiently managed, for instance when patients are improperly assisted to comprehensive their total program of treatment; when health-care furnishers prescribe the erroneous treatment, or the incorrect dose, or for too short a period of time; when the provider of medications to the clinics dispensing medications is irregular; or when the medications are of meager fantabulous. ${ }^{46-52}$

\section{Conclusion}

Genetic examinations which determine medication resistance to rifampicin with $>95 \%$ authentic are extremely indicative of MDR-TB; $<10 \%$ of rifampicin resistance is monoresistant, and so rifampicin resistance is a marker for MDR-TB in $>90 \%$ of cases. Because of its escalating frequency MDR-TB is recently subdivided into 'fundamental' MDR-TB, with resistance solely to rifampicin and isoniazid, and 'MDR-TB-addendum', with a identical resistance figure but with resistance to lone or greater supplemental $1^{\text {st }}$-line and/ or $2^{\text {nd }}$-line medications. MDR-TB is caused by bacteria that do not answered to, slightest, isoniazid and rifampicin, the dual consummate influential anti-TB medications. Patients with MDR-TB or RRTB necessitate treatment with $2^{\text {nd }}$ line treatment regimens, which are further sophisticated than those used to treat patients without MDR-TB. XDR-TB is a figure of MDR-TB which is also resistant to dual classes of $2^{\text {nd }}$-line anti-TB medications, creating it further sophisticated to treat. Symptoms of XDRTB are no otherness from usually or medication liable TB. Treatment failure happens when medications are malapropos prescribed owing to dearth of furnisher wisdom or owing to medication scarceness's. Occasionally the patient perhaps end $1^{\text {st }}$ line treatment owing to weaken side effects, dearth of an assistance network, incapability to remove time from function to acquire the treatment s/he seeks or distinctive rationale. Contradictory 
to what multiplex people credent, it is seldom for medication resistance to be caused by the willing ignorance of patients to regulate treatment.

\section{Data sources}

Sources searched include Google Scholar, Research Gate, PubMed, NCBI, NDSS, PMID, PMCID, and Cochrane database. Search terms included: multi drug-resistant tuberculosis, and extensively drug resistance tuberculosis.

\section{Acknowledgments}

The authors acknowledged Endnote-8, Google scholar, Medscape, Wikipedia, and PubMed.

\section{Funding}

None.

\section{References}

1. Sanchez JL, Sanchez JL, Cooper MJ, et al. Tuberculosis as a force health protection threat to the United States military. A review Mil Med. 2015;180(3):276-84.

2. World Health Organization (WHO). Companion handbook to the WHO guidelines for the programmatic management of drug-resistant tuberculosis. WHO/ HTM/TB/2014.11 WHO. Global Tuberculosis Report. 2014.

3. J. González Martín, JM García García, L Anibarro, et al . Documento de consenso sobre diagnóstico, tratamiento y prevención de la tuberculosis. Arch Bronconeumol. 2010; 46(5).255-274.

4. World Health Organization (WHO) Global Tuberculosis Report compiles data from 200 countries, monitoring the scale and direction of TB epidemics, implementation and impact of the Stop TB Strategy, and progress towards the Millennium Development Goals:

5. A special supplement to the Global Tuberculosis Report titled, Drugresistant TB-Surveillance \& Response, marks the 20th anniversary of the Global Project on Anti-Tuberculosis Drug Resistance Surveillance and its TB Supranational Reference Laboratory Network 2014.

6. Centers for Disease Control and Prevention. Provisional CDC guidelines for the use and safety monitoring of bedaquiline fumarate (Situro) for the treatment of multidrug-resistant tuberculosis. MMWR. 2013;62(9):1-12.

7. Jahnavi G, Sudha CH. Randomised controlled trial of food supplements in patients with newly diagnosed tuberculosis and wasting. Singapore Med J. 2010;51(12):957-962.

8. Partners in Health. The PIH Guide to the medical management of multidrug-resistant tuberculosis, 2nd Edition. Boston, MA. USAID TB CARE II. 2013.

9. National tuberculosis controllers association and national TB nursing consultant coalition. tuberculosis nursing: A comprehensive guide to patient care. atlanta: the national tuberculosis controllers association and national tuberculosis nursing consultant coalition. 2nd Edition. 2011.

10. Vincent N. Voluntary madness: My year lost and found in the loony bin. Penguin. 2008.

11. Caminero JA, Sotgiu G, Zumla A,et al. Best drug treatment for multidrugresistant and extensively drug-resistant tuberculosis. Lancet Infect Dis. 2010;10(9):621-629.

12. Chan ED, Laurel V, Strand MJ, et al. Treatment and outcome analysis of 205 patients with multidrug-resistant tuberculosis. Am J Respir Crit Care Med. 2004;169(10): 1103-1109.

13. Bereda G, Bereda G. Determinant of and factors influencing medication poor adherence to pulmonary tuberculosis treatment at the tuberculosis clinic of mettu karl referral hospital, southwestern oromia, ethiopia: A prospective cross sectional study, 2021. Archives in Respiratory \& Pulmonary Medicine. 2021;1(1).
14. Centers for disease control and prevention. provisional CDC guidelines for the use and safety monitoring of bedaquiline fumarate (Situro) for the treatment of multidrug-resistant tuberculosis. MMWR. 2013;62(9):1-12.

15. World Health Organization. Global tuberculosis report 2013. Geneva Switzerland: World Health Organization; 2013.

16. World Health Organization. Multidrug and extensively drug-resistant TB (M/XDR-TB): 2010 global report on surveillance and response. Geneva Switzerland. 2010.

17. Jindani AN, Enarson DA. Two 8-month regimens of chemotherapy for treatment of newly diagnosed pulmonary tuberculosis: international multi centrer and omised trial. Lancet. 2004; 8(364):1244-1251.

18. Moonan PK, Teeter LD, Salcedo K, et al. Transmission of multidrugresistant tuberculosis in the USA: a cross-sectional study. Lancet Infect Dis. 2013;13(9):777-784.

19. Royce S, Falzon D, van Weezenbeek C, et al. Multidrug resistance in new tuberculosis patients: burden and implications. Int J Tuberc Lung Dis. 2013;17(4):511-513.

20. Taylor AB, Kurbatova EV, Cegielski JP. Prevalence of anti-tuberculosis drug resistance in foreign-born tuberculosis cases in the U.S. and in their countries of origin. PLOS ONE. 2012;7(11):e49355.

21. World Care Council. Patients' charter for tuberculosis care. 2006.

22. World Health Organization. Drug-resistant TB surveillance \& response. Supplement: Global Tuberculosis Report. 2014.

23. World Health Organization. Global tuberculosis report. 2015

24. Berrada ZL, Lin SY, Rodwell TC, et al. Rifabutin and rifampin resistance levels and associated rpob mutations in clinical isolates of mycobacterium tuberculosis complex. Diagn Microbiol Infect Dis. 2016;85(2):177-181.

25. Centers for Disease control and prevention. availability of an assay for detecting mycobacterium tuberculosis, including rifampin resistant strains, and considerations for its use-United States, 2013. MMWR. 2013;62(41);821-824.

26. Centers for Disease Control and Prevention. Updated guidelines for the use of nucleic acid amplification tests in the diagnosis of tuberculosis. MMWR. 2009; 58(01):7-10.

27. CDC. Tuberculosis information for international travelers.

28. CDC. Extensively drug-resistant tuberculosis information.

29. CDC. CDC's Role in preventing XDR TB.

30. Cohen T, Sommers B, Murray M. The effect of drug resistance on the fitness of Mycobacterium tuberculosis. Lancet Infect Dis. 2003;3(1):13-21.

31. Luciani F, Sisson SA, Jiang H, et al. The epidemiological fitness cost of drug resistance in Mycobacterium tuberculosis. Proc Natl Acad Sci USA. $2009 ; 106(34): 14711-14715$.

32. Drobniewski F, Balabanova Y, Ruddy M, et al. Rifampin- and multidrugresistant tuberculosis in Russian civilians and prison inmates: dominance of the Beijing strain family. Emerg Infect Dis. 2002; 8(11):1320-1326.

33. Spradling P, Nemtsova E, Aptekar T, et al. Anti-tuberculosis drug resistance in community and prison patients, Orel Oblast, Russian Federation. Int J Tuberc Lung Dis. 2002;6(9):757-762.

34. Pfyffer GE, Strassle A, van Gorkum T, et al. Multidrug-resistant tuberculosis in prison inmates, Azerbaijan. Emerg Infect Dis. 2001;7(5):855-861.

35. Whyte T, Hanahoe B, Collins T,et al. Evaluation of the BACTEC MGIT 960 and $\mathrm{MB} \mathrm{BAC} / \mathrm{T}$ systems for routine detection of Mycobacterium tuberculosis. J Clin Microbiol. 2000 ;38(8):3131-3132.

36. Ruru Y, Matasik M, Oktavian A, et al. Factors associated with nonadherence during tuberculosis treatment among patients treated with DOTS strategy in Jayapura, Papua Province, Indonesia. Glob Health Action. 2018;11(1):1510592. 
37. Piatek AS, Telenti A, Murray MR, et al. Genetotypic analysis of Mycobacterium tuberculosis in two distinct populations using molecular beacons: implications for rapidsusceptibility testing. Antimicob Agents Chemother. 2000;44(1):103-10.

38. Espinal MA, Laszlo A, Simonsen L, et al. Global trends in the resistance to antituberculosis drugs. N Engl J Med. 2001;344(17):1294-1303.

39. Bwanga F, Joloba ML, Haile M,et al. Evaluation of seven tests for the rapid detection of multidrug-resistant tuberculosis in Uganda. Int J Tuberc Lung Dis. 2010;14(7):890-895.

40. Johnston JC, Shahidi NC, Sadatsafavi M, et al. Treatment outcomes of multi drug resistant tuberculosis: a systematic review and metaanalysis. PLoS One. 2009;4(9):e6914.

41. Multidrug and extensively drug-resistant TB (M/ XDR-TB): 2010 global report on surveillance and response. Geneva: World Health Organization. 2010 .

42. Extensively drug-resistant tuberculosis (XDRTB): recommendations for prevention and control. Wkly Epidemiol Rec. 2006;81(45):430-432.

43. Gandhi NR, Moll A, Sturm AW, et al. Extensively drug resistant tuberculosis as a cause of death in patients coinfected with tuberculosis and HIV in a rural area of South Africa. Lancet. 2006;368(9547):1575-1580.

44. Van der Werf MJ, Sprenger M. Drugresistance - a challenge for tuberculosis control in the European Union and European Economic Area. Euro Surveill. 2014;19(11):20737.

45. World Health Organization. Global tuberculosis report 2016. Geneva: WHO. 2016.
46. Migliori GB, Sotgiu G, Gandhi NR, et al. Drug resistance beyond extensively drug-resistant tuberculosis: individual patient data metaanalysis. Eur Respir J. 2013;42(1):169-179.

47. Gehre F, Otu J, Kendall L, et al. The emerging threat of pre-extensively drug-resistant tuberculosis in West Africa: preparing for large-scale tuberculosis research and drug resistance surveillance. BMC Med. 2016;14(1):160

48. Bakayoko AS, Ahui BJ, Kone Z, et al. Extensively drug resistant tuberculosis in Ivory Coast. Rev Pneumol Clin. 2015; 71(6):350-353.

49. Andrews JR, Shah NS, Gandhi N, et al. Multidrug-resistant and extensively drug-resistant tuberculosis: implications for the HIV epidemic and antiretroviral therapy rollout in South Africa. J Infect Dis. 2007;196(Suppl 3):S482-90.

50. Maiga M, Siddiqui S, Diallo S, et al. Failure to recognize nontuberculous mycobacteria leads to misdiagnosis of chronic pulmonary tuberculosis. PLoS ONE. 2012;7(5):e36902.

51. Coeck N, de Jong BC, Diels M, et al. Correlation of different phenotypic drug susceptibility testing methods for four fluoroquinolones in Mycobacterium tuberculosis. J Antimicrob Chemother. 2016;71(5):12331240 .

52. Lange C, Abubakar I, Alffenaar JW, et al. Management of patients with multidrug-resistant/extensively drug-resistant tuberculosis in Europe: a TBNET consensus statement. Eur Respir J. 2014;44(1):23-63. 